\title{
Transient Biometrics using Finger Nails
}

\author{
Igor Barros Barbosa Theoharis Theoharis Christian Schellewald \\ Department of Computer and Information Science \\ Norwegian University of Science and Technology \\ Cham Athwal \\ School of Digital Media Technology \\ Birmingham City University
}

\begin{abstract}
Transient biometrics, a new concept for biometric recognition, is introduced in this paper. A traditional perspective of biometric recognition systems concentrates on biometric characteristics that are as constant as possible (such as the eye retina), giving accuracy over time but at the same time resulting in resistance to their use for non-critical applications due to the possibility of misuse. In contrast, transient biometrics is based on biometric characteristics that do change over time aiming at increased acceptance in noncritical applications. We show that the fingernail is a transient biometric with a lifetime of approximately two months. Our evaluation datasets are available to the research community.
\end{abstract}

\section{Introduction}

Biometric recognition systems offer unique advantage when compared to conventional recognition systems, such as smart cards or passwords. By using a biometric recognition system, the subject does not need to carry or remember any id or password, and there is less risk of loss or disclosure of the recognition token. Biometric recognition is thus gaining support and acceptance in critical recognition situations supported by governments or other large organizations.

Despite the advantages of biometric recognition systems, a major concern of individuals is the possibility of misuse of their biometric data. A card or password can be canceled, but what happens if your biometric data falls into the wrong hands? An individual's privacy may be compromised (e.g. through their use for unauthorized recognition purposes) or discrimination may be enabled (e.g. through unauthorized use by insurance agents).

Cancelable biometrics $[7,8]$ attempts to answer this concern by pre-transforming (distorting) the biometric data be- fore the biometric signature is extracted. The transformation is non-reversible. Thus, the potential for misuse is limited by the fact that the misuser cannot retrieve the original biometric data, and the transformation can be changed at any time. However, cancelable biometrics requires that the subject trusts the biometrics capture point and also that the misuse is detected in order to activate a transform change.

There is plenty of scope for biometric recognition systems to become more socially acceptable, in the sense that society could accept and use such systems in day-to-day scenarios. The acceptability issue remains particularly open when dealing with non-critical scenarios and collaborative subjects. For instance, individuals will not happily offer their fingerprints just to have access to their hotel room. The points raised above limits the use of biometric technologies in a multitude of noncritical situations.

In this paper we introduce transient biometrics. Transient biometrics is defined as biometric recognition technologies which rely on biometric characteristics that are proven to change over time. Thus, they automatically cancel themselves out after a known period of time. A transient biometric approach for the verification task is shown in Fig. 1. In contrast to cancelable biometrics, it is the actual biometric data that are naturally changing over time. As a consequence it will presumptively help in the creation of more sociable acceptable recognitions systems. We show that images of the finger nail constitute a transient biometric with a lifetime of two months.

The remaining of the paper is organised as follows. Section 2 briefly presents the biometric literature which employs finger nails. Section 3 details our approach followed by Section 4 that shows experimental results. Finally, Section 5 concludes the paper, envisaging some future perspectives. 


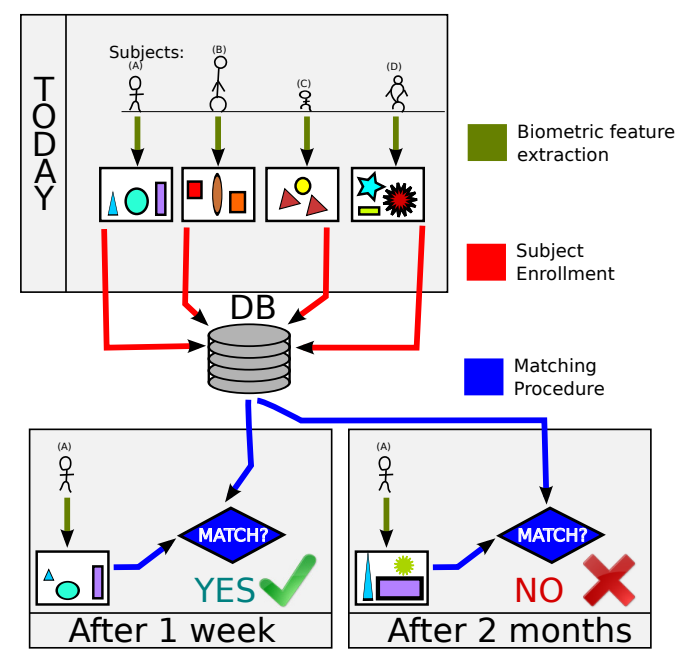

Figure 1. Example of a verification task employing transient biometrics.

\section{Previous Work}

The use of finger nails in biometrics applications has been the topic of a few different lines of research. A complex acquisition system employing tailored lighting equipment has been designed to acquire images of the nail bed, which is the skin under the nail plate [9]. Such images are then used for individual authentication by exploring features from the nail bed grooves. This is possible because the nail bed is unique to each individual [3].

Recently, a cancelable biometric approach has developed stickers, which can be placed over finger nails for an identification process [4]. In the presented paper, thumb images were acquired against a black background, for it made it easier to compute the boundaries of the thumb. The stickers glued over the fingernails provide two landmarks which are used in the feature extraction process. The finger outline is pursued, and the distance from the outline to the landmarks creates a distance profile. The final matching procedure is done by computing correlation coefficients between distance profiles. Whenever such sticker is removed or repositioned over the finger nail, the distance profile changes. Therefore, it is possible to cancel the biometric by replacing the landmarks or by shifting the sticker position. The finger nail would only play a role in the recognition system if the nail outgrew the finger. Although it is a valid cancelable biometric approach, this work does not truly explore finger nails for biometric recognition.

The information of the nail surface has been explored by a biometric authentication system proposed in [2]. This approach uses images of hands in order to extract information from the finger nails. First, the fingers are segmented by a smart contour segmentation algorithm, then the nails are segmented by grey scale thresholding. This simple segmentation approach is likely to work given that the employed dataset was biased with respect to subject's skin tones. The individual authentication process is built upon the hamming distance of high frequency Haar wavelet coefficients. Experimental results show reasonable recognition rates using three sample images per subject. The first two images are employed in training while the last image is used for testing. Despite the positive results, this work does not explore how the recognition rate behaves with respect to the growth of the finger nails; the authors do not provide the time difference between acquisitions, so we assume that all images were acquired on the same date.

\section{The Proposed Approach}

The transient biometric approach presented in this work addresses the identification problem. Therefore, our objective is to identify a subject by comparing a biometric signature against a dataset of previously collected samples. To this end, the proposed solution can be divided into three phases. The first phase deals with image segmentation and pre-processing. The second phase extracts the biometric signature, while the third phase addresses signature matching.

\subsection{Nail image pre-processing}

Images of the right index finger are the source of biometric information. Since the images were taken on different days and sometimes with different cameras, the preprocessing of input images is a key step for the overall process. It assures that the images delivered to the signature extraction algorithm fulfil the requisites regarding colour correction, nail plate registration and image size.

Pre-processing starts by segmenting the nail from the finger images. Such segmentation is done by an active shape model (ASM) see [11]. The active shape model requires a set of training images where the segmentation has been manually performed (contour drawn). The algorithm employs Principal Component Analysis ( $P C A$ ) to find eigen segmentation contours, with very accurate results. The ASM also describes the image around each control point with a grey-level appearance model. This grey-level appearance model is computed using lines perpendicular to each control point, and it is built using the first derivative of greylevel images. This appearance model will be used later in an iterative fashion to correct the position of control points while searching for the best segmentation contour.

The ASM requires training data and for this purpose the dataset $\boldsymbol{D} 0 \mathbf{1}$ was used. This training dataset represents the first acquisition day. It contains an image of the right index finger for every enrolled individual, with a total of $32 \mathrm{im}-$ ages (see more information on the dataset in Sec. 4). The ASM was trained with two main landmarks and 20 control points between them. The first landmark is placed at the base of the nail plate just by the intersection with the finger 
skin. Meanwhile, the other landmark is placed opposite to it, by the end of the nail plate. An input sample is shown as $[\mathrm{A}]$ in Fig. 2, and the resulting segmentation is shown as [B].

Image pre-processing continues by computing the bounding box. Next, the bounding box is converted to grey scale, making the input more robust to changes. These changes are likely to happen due to wrong white balance or even due to the use of different cameras. The overall pre-processed image is given by resizing the bounded box to a width and height of 128 pixels. The resulting image is shown as $[\mathrm{C}]$ in Fig. 2

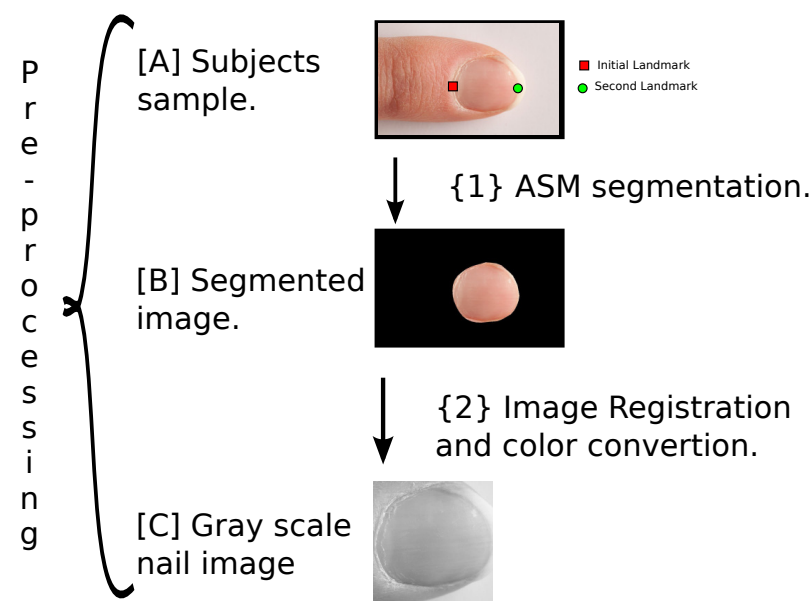

Figure 2. Sample results from the image pre-processing pipeline.

To make the training process more robust each image is used to create multiple variations. These are given through the application of Wiener Filters, by shifting the segmented region-of-interest by a few pixels and by the application of histogram equalisation. When all these images modification are combined in a chain, every input image generates 810 variations.

\subsection{Signature extraction based on uniform LBP}

It has been observed that a nail plate is categorised by a unique texture which is influenced by patterns in the nail bed [3]. The nail plate texture is also dependent on interaction with external factors. Hence, it is common to notice white spots and marks originating from scratches or bumps. Since the nail plate possesses such rich texture, we have opted to base signature extraction on Local Binary Patterns $(L B P)$, which is a successful and robust texture descriptor [5]. LBPs are known for their computational efficiency and their capacity to discriminate micro-patterns. They have also been successfully employed in a wide variety of applications, ranging from texture classification [6] (their original purpose), to facial recognition [1]. Thus, $L B P$ has been selected for the signature extraction process.
$L B P$ are computed pixel wise, relying on the pixel neighbourhood information. The computation starts by defining a neighbouring circle with a radius of $R$ pixels and $P$ evenly spaced sample points. Bilinear interpolation is used to compute the value of a sample point if it does not fall on a pixel center. Fig. 3 illustrates two possible circular neighbourhoods. $L B P$ is computed for the pixel $g_{c}$, located in the center of the circle, using the threshold operation of Eq. 1.
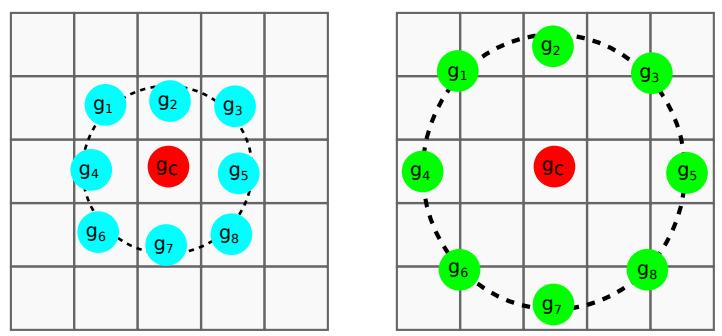

Figure 3. Sample neighbourhoods of $(P, R)=(8,1)$ and $(P, R)=(8,2)$.In these examples $g_{p}$ are the sample points, where $p$ ranges from 1 to $P$.

$$
\begin{array}{r}
L P B_{P, R}=\sum_{p=1}^{P} \phi\left(g_{p}-g_{c}\right) \times 2^{p-1} \\
\phi(x)=\left\{\begin{array}{lll}
1 & \text { if } & x \geq 0 \\
0 & \text { if } & x<0
\end{array}\right.
\end{array}
$$

An $L B P$ is uniform whenever the coded value is composed of zero, one or two bit-wise transitions. Thus, the patterns 11111111 and 00001111 are uniform since they have zero and two bit-wise transitions, respectively. On the other hand, the pattern 10101010 is non-uniform since it is composed of eight transitions. If a coded pixel uses eight sample points, it is possible to generate 256 patterns, out of which 58 are called uniform LBP patterns. The work of $[1,6]$ confirms that uniform Local Binary Patterns $\left(L B P^{u 2}\right)$ account for the vast majority of encountered patterns. Therefore, signature extraction employs uniform $L B P$ for describing the nail plate texture.

The signature extraction process starts by dividing the pre-processed nail plate image into smaller image blocks. A $4 \times 4$ grid is used for the division, generating 16 blocks of $32 \times 32$ pixels. A histogram of the values of $L B P_{8,2}^{u 2}$ is then computed for each block. The histogram is composed of 59 bins, 58 of them used for uniform patterns and the last bin for non-uniform ones. The signature is then created by concatenating the 16 histograms, thus forming a global descriptor of the nail plate. This process is illustrated in Fig. 4 and follows the methodology proposed in [1]. A descriptor capable of describing texture and its spatial relationships is thus created, which is very suitable for the nail plate. However, the resulting signature has 944 features. The dimensionality curse is avoided by the use of Karhunen-Loeve 
Analysis. The data from $\boldsymbol{D 0 1}$ (see Sec. 4), is analysed by Karhunen-Loeve decomposition which allows us to map the extracted signature into a subspace of 200 dimensions.

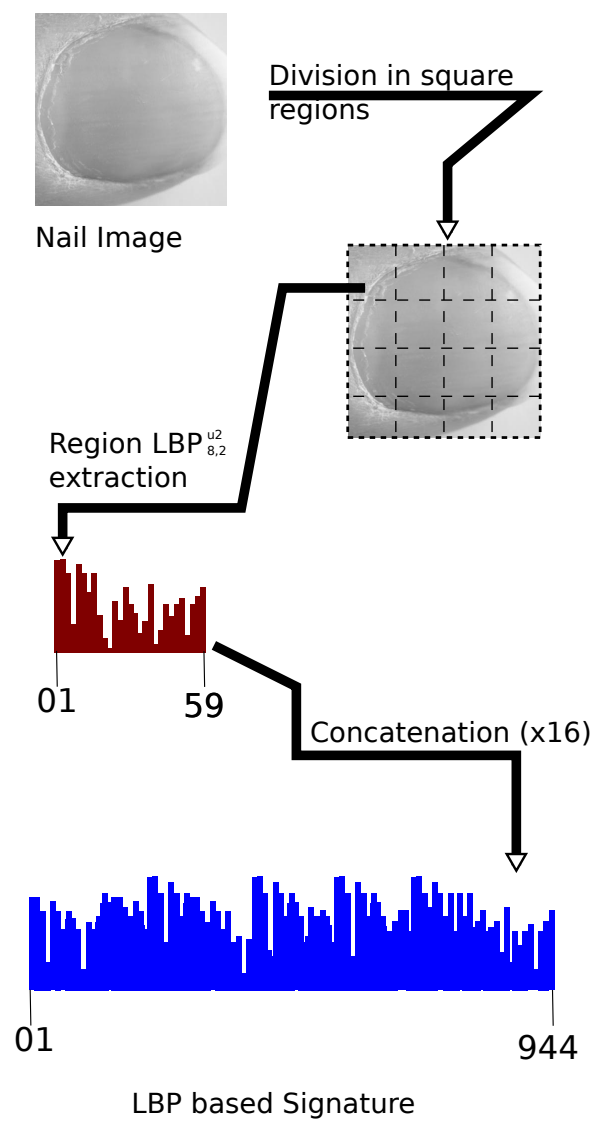

Figure 4. Signature extraction pipeline

\subsection{Signature matching}

Signature matching essentially identifies patterns within a dataset. These patterns should ideally have small variation between objects of the same class (e.g. nail images of the same subject) while they should have large variation across different classes. To identify such patterns Bayesian classification is employed. A Bayesian classifier estimates the boundaries between classes assuring that the Bayes risk/error is minimal. The Bayes rule states that the probability of a subject belonging to a class $\omega_{k}$ given an observation $\boldsymbol{z}$ (signature after dimensionality reduction) is given by the posterior probability as shown in Eq. 2 .

$$
P\left(\omega_{k} \mid \boldsymbol{z}\right)=\frac{p\left(\boldsymbol{z} \mid \omega_{k}\right) P\left(\omega_{k}\right)}{p(\boldsymbol{z})} .
$$

where $P\left(\omega_{k} \mid \boldsymbol{z}\right)$ is the posterior probability, $p\left(\boldsymbol{z} \mid \omega_{k}\right)$ is the probability distribution of $\boldsymbol{z}$ coming from a subject with known class $\omega_{k}, P\left(\omega_{k}\right)$ is the prior probability of having the class $\omega_{k}$ and $p(\boldsymbol{z})$ is the distribution of the observation.
If a unitary cost is assumed for every wrong classification, the minimisation of the Bayes risk becomes equivalent to the maximisation of posterior probability [10]. Therefore, the classifier can be re-written as

$$
\hat{\omega}_{\text {map }}(\boldsymbol{z})=\underset{j}{\operatorname{argmax}}\left\{p\left(\boldsymbol{z} \mid \omega_{j}\right) P\left(\omega_{j}\right)\right\} .
$$

Our assumption is that the conditional probability density function, $p\left(\boldsymbol{z} \mid \omega_{j}\right)$, can be modelled as normal. Therefore the observations are assumed to have an expectation vector $\boldsymbol{\mu}_{k}$ and a covariance matrix $\boldsymbol{C}_{k}$, yielding to the function shown in Eq. 4

$$
p\left(\boldsymbol{z} \mid \omega_{j}\right)=\frac{1}{\sqrt{(2 \pi)^{N}\left|C_{k}\right|}} e^{\left(\frac{-\left(\boldsymbol{z}-\mu_{k}\right)^{T} C_{k}^{-1}\left(\boldsymbol{z}-\mu_{k}\right)}{2}\right)}
$$

Such a conditional probability density function results in a quadratic classifier. It was determined experimentally that if we assume that the covariance does not depend on the class, e.g. $C_{k}=C$ for all possible classes, we end up with a linear Bayes normal classifier which outperformes the quadratic classifier.

The linear Bayes normal classifier is them applied to every computed $z$ and the final classification is given by the conjunction of the 810 images which were artificially created. To get the final classification of each input image, a final distance measure is created by Eq. 5 .

$$
D\left(\boldsymbol{Z}^{810}, \omega_{k}\right)=\sum_{n=1}^{810}\left|\log _{e} c\left(\boldsymbol{Z}_{n}^{810}, \omega_{k}\right)\right|
$$

where $Z^{810}$ represents the conjunction of 810 observations derived from a single input image, $c\left(\boldsymbol{Z}_{n}^{810}, \omega_{k}\right)$ represents the confidence of the nth observation of $Z$ being from class $\omega_{k}$. When this distance measure is employed, the most probable class is given by the smallest computed distance.

\section{Experiments}

This section will describe the experimental dataset and the effect of nail plate growth on identification performance.

\subsection{Dataset Creation}

Our dataset is composed of three different sets of data. All three sets have followed the same acquisition process. First the right index finger of the subject is placed over a white sheet of paper, which is supported by a flat surface. The finger is placed over this surface without putting pressure against it, as pressure changes the colour of the finger nail. Then a diffuse light is placed so the light source points to the top of the finger. Therefore, the finger is virtually pointing to the light source. Such lighting condition avoid highlights and help achieve proper exposure. Finally the 
image is acquired by framing only the contents of the white paper and maintaining proper focus on the finger nail plate.

Two things differentiate the three sets of data: acquisition date and number of subjects. Set $\mathbf{D 0 1}$ consists of the collection of images from the first acquisition day. Not surprisingly, this represents the largest set in terms of number of subjects, consisting of data from 32 individuals. The second set $\mathbf{D 0 8}$ is composed of images acquired seven days after the initial acquisition. This set is composed of 24 individuals who were also part of D01. The third and smallest set, D70, contains images of 17 subjects, all part of D08. The images of this third set where acquired seventy days after the initial acquisition day. Figure 5 shows samples of four individuals who were represented in all three datasets. The nail dataset and its future extensions will be made available at NTNU's Visual Computing group website [http://www.idi.ntnu.no/grupper/vis/].

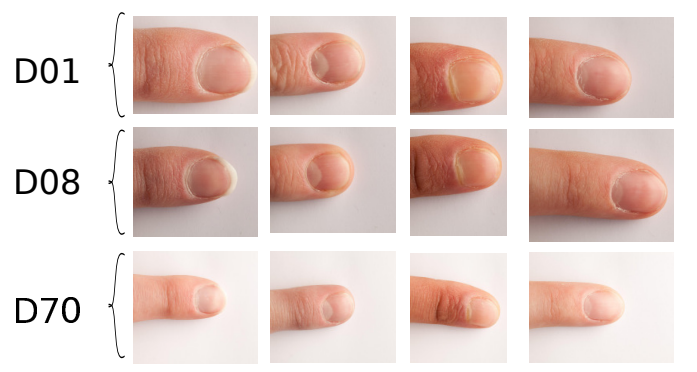

Figure 5. Images samples from available datasets. Each Row represents a dataset while each column represents a different subject.

\subsection{Identification performance analysis}

In all experiments the classifier was trained using only information from D01. The classifier was then applied to $D 08$ and $D 70$ to evaluate the decay of identification performance, as expected for a transient biometric solution. As D70 contains only 17 subjects, the classifier $K_{17}$ is trained on D01 using only the information from subjects available in D70. The classifier is then applied to $\mathbf{D 0 8}$ and $\mathbf{D 7 0}$. The cumulative matching curve $(C M C)$ is used as a standardised evaluation graph. It assesses the classification performance in identification problems. $C M C$ models the probability of a signature from a test dataset, in this case $\mathbf{D 0 8}$ and $\mathbf{D 7 0}$, being correctly matched in the first $P$ ranked subjects from the training dataset $\mathbf{D 0 1}$. Such rank is derived from the computed distances, as specified in Section 3.3. The CMC curve for both $\mathbf{D 0 8}$ and $\boldsymbol{D 7 0}$ are plotted in Fig. 6. The performance decay observed in $\boldsymbol{D 7 0}$ is evidence that the biometric signature extracted from the nail plate biometric is of short persistence. Thus the nail plate is a good candidate for transient biometric solutions. If the normalised area under the curve is taken as a performance measure, the changes in the nail plate during the 62 days between the acquisition of $\boldsymbol{D 7 0}$ and $\mathbf{D 0 8}$ account for a $9.32 \%$ decay. If rank one recognition is taken as a measure of performance, the results are even more conclusive: the two month interval represents a decay of $58.82 \%$ in the probability of identifying the individual in a first guess.

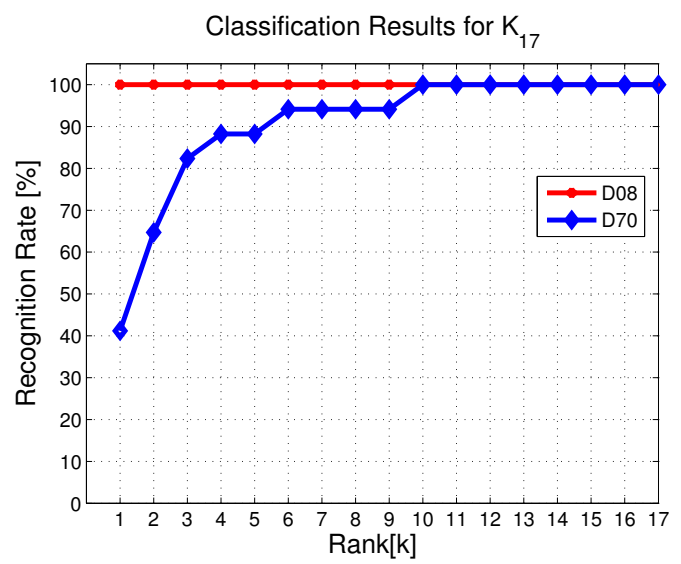

Figure 6. Cumulative matching curves for classifier $K_{17}$ evaluated on $\mathbf{D 0 8}$ and D70. The classifier was trained for 17 subjects and then applied to images acquired 8 days later (D08) and 70 days later (D70). The decay in performance from D08 to D70 arguments in favour that finger nails are transient biometrics. Therefore, the biometric information changes during time, making the identification process unreliable after two months.

A second classifier $K_{24}$ was trained on D01 using only the information from subjects available in D08. This classifier is composed of 24 subjects and represents a harder classification task than the one $K_{17}$ was assigned. The objective was to show that positive identification is possible with nail biometrics over a short period of time. The classification results are shown as a $C M C$ curve in Fig. 7.

Finally, Table 1 summarises the results of the three classification problems presented.

\begin{tabular}{l|lcr}
\hline \multicolumn{4}{c}{ Classifier $K_{17}$} \\
\hline Test Dataset & nAUC & Rank 1 & Rank 2 \\
\hline D08 & $100.00 \%$ & $17 / 17$ & $17 / 17$ \\
D70 & $90.657 \%$ & $7 / 17$ & $11 / 17$ \\
\hline \multicolumn{4}{c}{ Classifier $K_{24}$} \\
\hline D08 & $99.479 \%$ & $22 / 24$ & $23 / 24$ \\
\hline
\end{tabular}

Table 1. Classification performance

\section{Conclusion}

So far, biometrics research has produced significant results in terms of universality, distinctiveness and permanence. Acceptability still remains as an important issue and the main reason behind this is the fear of misuse of one's 


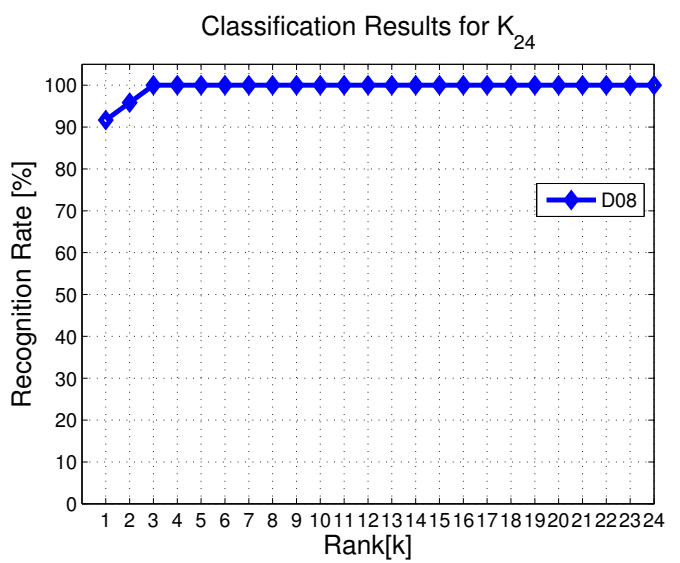

Figure 7. Cumulative matching curves for classifier $K_{24}$ evaluated on D08. The classifier was trained for 24 subjects and then applied to images acquired 8 days later (D08). The overall performance achieved in D08 arguments in favour that finger nails are transient biometrics which a week interval has little effect in the recognition capabilities.

permanent biometric data. Individuals are thus reluctant to volunteer their biometric characteristics where possible, and the leap in usability that biometric technology offers (i.e. password- and devicefree access to resources), cannot be realised.

This work introduces a new idea to address the acceptability issue inherent to biometric solutions. This approach, designed for collaborative individuals, instead of recording permanent data, records transient data, i.e. data that do change over time and are thus cancelled by nature. Users, who know that the biometric data they offer is going to be useless for recognition purposes after a certain amount of time, are likely to be more willing to offer it, even for dayto-day applications. This approach is termed transient biometrics; the idea is to use features with a short permanence, giving a diminutive period of recognition.

A transient biometric solution to the identification task was presented, which exploits texture features, extracted from finger nail images, investigating different acquisition intervals. Identification performance was high within a week but degraded considerable after a two month period. This indicates that finger nail images are a valid transient biometric solution.

In subsequent work we intend to expand our dataset with more subjects, more realistic capture conditions and different skin tones.

\section{References}

[1] T. Ahonen, A. Hadid, and M. Pietikäinen. Face description with local binary patterns: application to face recognition. IEEE transactions on pattern analysis and machine intelligence, 28(12):2037-41, Dec. 2006.
[2] S. Garg, A. Kumar, and M. Hanmandlu. Biometric authentication using finger nail surface. In 2012 12th International Conference on Intelligent Systems Design and Applications (ISDA), pages 497-502. IEEE, Nov. 2012.

[3] R. Krstic. Human Microscopic Anatomy: An Atlas for Students of Medicine and Biology. Springer, 1991.

[4] N. Nishiuchi and H. Soya. Cancelable Biometric Identification by Combining Biological Data with Artifacts. In 2011 International Conference on Biometrics and Kansei Engineering, number ii, pages 61-64. IEEE, Sept. 2011.

[5] T. Ojala, M. Pietikäinen, and D. Harwood. A comparative study of texture measures with classification based on featured distributions. Pattern Recognition, 29(1):51-59, Jan. 1996.

[6] T. Ojala, M. Pietikainen, and T. Maenpaa. Multiresolution gray-scale and rotation invariant texture classification with local binary patterns. IEEE Transactions on Pattern Analysis and Machine Intelligence, 24(7):971-987, July 2002.

[7] N. K. Ratha, J. H. Connell, and R. M. Bolle. Enhancing security and privacy in biometrics-based authentication systems. IBM Systems Journal, 40(3):614-634, 2001.

[8] C. Rathgeb and A. Uhl. A survey on biometric cryptosystems and cancelable biometrics. EURASIP Journal on Information Security, 2011(1):3, 2011.

[9] A. Topping, V. Kuperschmidt, and A. Gormley. United States Patent US005751835A, 1998.

[10] F. van der Heijden, R. P. W. Duin, D. de Ridder, and D. M. J. Tax. Classification, parameter estimation and state estimation - an engineering approach using Matlab. John Wiley \& Sons, Chichester, 2004.

[11] B. van Ginneken, A. F. Frangi, J. J. Staal, B. M. ter Haar Romeny, and M. A. Viergever. Active shape model segmentation with optimal features. IEEE transactions on medical imaging, 21(8):924-33, Aug. 2002. 